\title{
Initial Results from the Donner 600 Crystal Positron Tomograph*
}

\author{
S.E. Derenzo, R.H. Huesman, J.L. Cahoon, \\ A. Geyer, D. Uber, T. Vuletich, and T.F. Budinger \\ Donner Laboratory and Lawrence Berkeley Laboratory \\ University of California \\ Berkeley, CA 94720
}

LBL- -22363

DE88 006343

\section{Abstract}

We describe a positron tomograph using a single ring of 600 close-packed 3 $\mathrm{mm}$ wide bismuth germanate (BGO) crystals coupled to $14 \mathrm{~mm}$ phototubes. The phototube preamplifier circuit derives a timing pulse from the first photoelectron, and sends it to address and coincidence circuits only if the integrated pulse height is within a pre-set window. The timing delays and pulse height windows for all 600 detectors and the coincidence timing windows are computer adjustable. An orbiting positron source is used for transmission measurements and a look-up table is used to reject scattered and random coincidences that do not pass through the source. Data can be acquired using a stationary mode for $1.57 \mathrm{~mm}$ lateral sampling or the two-position clam sampling mode for $0.79 \mathrm{~mm}$ lateral sampling. High maximum data rates are provided by 45 parallel coincidence circuits and 4 parallel histogram memory units. With two-position sampling and $1.57 \mathrm{~mm}$ bins, the reconstructed point spread function (PSF) of a $0.35 \mathrm{~mm}$ diam ${ }^{22} \mathrm{Na}$ wire source at the center of the tomograph is circular with $2.9 \mathrm{~mm}$ full-width at half-maximum (fwhm) and the PSF at a distance of $8 \mathrm{~cm}$ from the center is elliptical with a radial fwhm of 4.0 $\mathrm{mm}$ and tangential fwhm of $3.0 \mathrm{~mm}$.

*Presented at the IEEE Nuclear Science Symposium, Washington, D.C., October 1986. To be published in the IEEE Nuclear Science Transactions, Volume NS-34, 1987.

This work was supported in part by the U.S. Department of Energy, under Contract No. DE-AC03-76SF00098, and in part by Public Health Service Grant Nos. P01 HL25840 and R01 CA38086. 


\section{Introduction}

There is considerable interest in improving the spatial resolution in positron emission tomography for the brain and neck, because many important functional regions are too omall to be investigated quantitatively with $10-20 \mathrm{~mm}$ resolution. For example, a spatial resolution of 2 to $3 \mathrm{num}$ fwhm will permit measurement of the dynamics of blood flow in the cerebral arteries and the uptake and disappearance of labeled tracers in small, functionally distinct brain nuclei.

This work shows that $3 \mathrm{~mm}$-wide BGO crystals can provide excellent resolution with good efficiency, in spite of factors such as the range of positrons in tissue, deviations from $180^{\circ}$ emission, off-axis penetration through the crystals, and Compton scattering resulting in multiple crystal interactions. This tomograph is designed for quantitation of tracer activity in $5 \mathrm{~mm}$ regions, which requires a spatial resolution of $2-3 \mathrm{~mm}$, and this in turn requires sampling the projections with a frequency of one per $\mathrm{mm}$ or finer.

Other work to improve spatial resolution includes the multi-crystal analog system of Burnham and Brownell which has a spatial resolution of $4.5 \mathrm{~mm} \mathrm{fwhm}^{\mathrm{l}}$, the small animal system of Tomitani et al which has a resolution of $3.5 \mathrm{~mm} \mathrm{fwhm}^{3}$, and the ECAT III whole body positron tomograph which has a spatial resolution of $5 \mathrm{~mm}$ fwhm.

\section{Detectors and Gantry}

Each BGO crystal is individually coupled to a $14 \mathrm{~mm}$ diam phototube (Figure 1, Table 1). The BGO crystals have three different shapes, depending on the orientation of the associated phototube. These shapes were chosen experimentaily to maximize the light output and pulse height resolution. Of the 600 crystals, 240 are $10 \mathrm{~mm} \times 3 \mathrm{~mm} \times 30 \mathrm{~mm}$ deep with a $45^{\circ}$ cut (crystals $A$ and $E$ in Figure 1), 240 are $10 \mathrm{~mm} \times 3 \mathrm{~mm} \times 23 \mathrm{~mm}$ deep (crystals $B$ and D in Figure 1), and 120 are $10 \mathrm{~mm} \times 3 \mathrm{~mm} \times 30 \mathrm{~mm}$ deep (crystal $\mathrm{C}$ in Figure 1). Only certain regions of the crystals are coupled to the phototubes and the other crystal surfaces are polished and coated with dry $\mathrm{MgO}$ reflector. The reflector coating between the crystals is thin, and the center-to-center crystal spacing is $3.14 \mathrm{~mm}$. Since the phototubes are coupled to 3 sides of the crystal array, this approach is limited to a single ring of crystals. The detectors have a pulse height resolution of about $25 \%$ fwhm for 511 $\mathrm{keV}$ annihilation photons. 
Figure 1: Sketch of detector configuration. Each BGO crystal is coupled indiviudally to a $14 \mathrm{~mm}$ diā̄is phoutotube. Crystals $\mathrm{A}$ and $\mathrm{E}$ are $30 \mathrm{~mm}$ long, $10 \mathrm{~mm}$ high, and $3 \mathrm{~mm}$ thick, cut at a 45 deg angle to improve light collection efficiency. Crystals $B$ and $D$ are $23 \mathrm{~mm}$ long, $10 \mathrm{~mm}$ high, and 3 $\mathrm{mm}$ thick. Crystal C is 30 $\mathrm{mm}$ long, $10 \mathrm{~mm}$ high, and $3 \mathrm{~mm}$ thick.

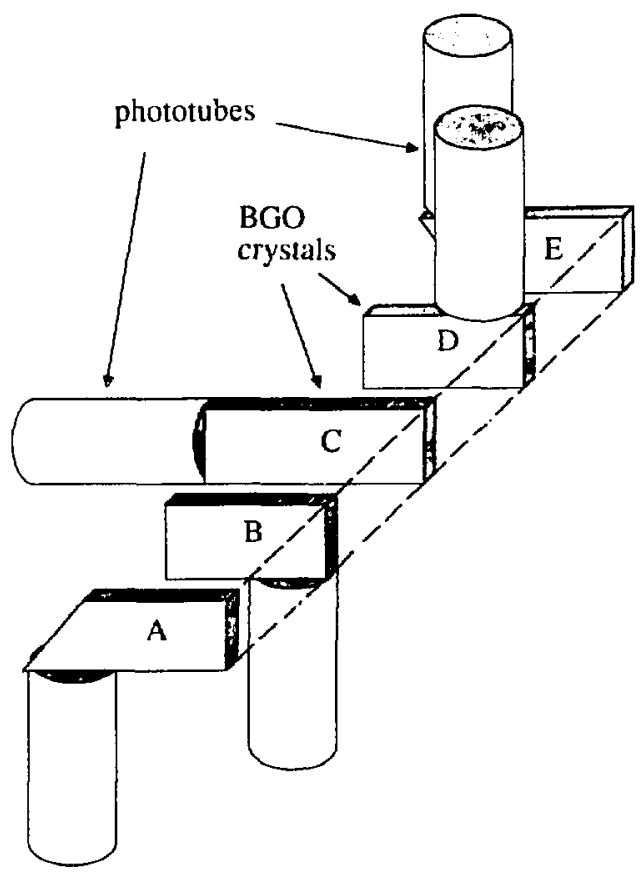

TABLE 1: DESCRIPTION OF TOMOGRAPH

Number of BGO crystals Detector ring diameter Patient port diameter BGO crystal size

BGO crystal c-c spacing Number of sampling positions Number of projection data
600

$60 \mathrm{~cm}$

$30 \mathrm{~cm}$

$3 \mathrm{~mm} \times 10 \mathrm{~mm}$

$x 23$ to $30 \mathrm{~mm}$ deep

$3.14 \mathrm{~mm}$

2

120,000 
The lead shielding has a gap that is adjustable from $1 \mathrm{~mm}$ to $10 \mathrm{~mm}$, an inner diameter of $30 \mathrm{~cm}$, and an outer diameter of $60 \mathrm{~cm}$. The section thickness is $5 \mathrm{~mm}$, corresponding to the geometric axial response.

The detectors are mounted on two $\mathrm{C}$-shaped arms that are hinged together at one point on the detector ring. A cam mechanism, opposite the hinge, can open the arms $3.14 \mathrm{~mm}$, which is the detector center-to-center spacing. When the arms are shut, the detector ring forms a circle of 600 close-packed detectors. When the arms are opened, the detector ring approximates a 601 crystal ring with one detector missing. The combined data set provides 120,000 projection measurements with 100 rays at each of 1200 angles. Lateral spacing is staggered in angle, which results in a linear sampling of $0.79 \mathrm{~mm}$, which is one-fourth of the center-to center crystal spacing. ${ }^{5}$

\section{Electronics}

The anode signal from each phototube is sent to its own circuit via coaxial cable. This circuit amplifies the signal, integrates the charge for pulse height discrimination, and starts a timing cycle from the first photoelectron iiberated by the photocathode. ${ }^{8,7}$ Two switch-selectable event vetos are provided, which reset the timing cycle under the following conditions: (1) the photoelectron pulse is not followed by subsequent pulses within 50 nsec (indicating a noise pulse), or (2) a pulse height threshold is exceeded in either the left or right adjacent channisl (indicating that the annihilation photon has interacted in more than one crystal). Note that in $\mathrm{BGO}$, the first interaction of a $511 \mathrm{keV}$ annihilation photon is by Compton scattering $53 \%$ of the time and by photoelectric absorption $47 \%$ of the time.

The amount of integrated charge is compared to upper and lower discriminators whose thresholds are set by computer control. A valid $511 \mathrm{keV}$ detection requires a pulse height corresponding to an energy of between $350 \mathrm{keV}$ and 1000 $\mathrm{keV}$ in one crystal. If the output of the charge integrator is within the pulse height window, and no vetos have caused the timing cycle to be reset, the phototube circuit generates a timing pulse that is sent to address generation and coinciäence circuits.

The pulse height window for each integrator and the timing delay for each phototube are cumputer controlled by 1800 digital to analog converters. An additional 90 converters control the width of the coincidence windows in each coincidence circuit. Prior to each study, a computer program is used to sequence the con/erters to maximize the coincident count rates for the orbiting transmission source. 


\section{Results}

The detector pair timing resolution is 5 nsec fwhm and a 9 nsec full timing window accepts $>90 \%$ of the coincident events (Table 2). The photopeak resolution ranges from 20 to $30 \%$ fwhm. At the center of the gantry, a $0.35 \mathrm{~mm}$ diam ${ }^{22} \mathrm{Na}$ wire ${ }^{8}$ has a detector pair resolution of $2.4 \mathrm{~mm}$ fwhm (Figures 2 and 3), as determined by moving the source perpendicular to the line between the detectors in $0.2 \mathrm{~mm}$ steps. At distances of $8 \mathrm{~cm}$ and $12.5 \mathrm{~cm}$ from the center of the gantry, the crystal pair resolution is $3.9 \mathrm{~mm}$ fwhm and $5.0 \mathrm{~mm}$ fwhm, respectively.

Reconstructions were performed using the convolution method ${ }^{9}$ on $1.79 \mathrm{~mm}$ projection bins. The reconstructed images of the $0.35 \mathrm{~mm}$ diam ${ }^{22} \mathrm{Na}$ wire source at the center of the tomograph have a PSF that is circular with a fwhm of $2.9 \mathrm{~mm}$ (Figure 4). At a distance of $8 \mathrm{~cm}$ from the center of the gantry, the PSF is elliptical with a radial fwhm of $4.0 \mathrm{~mm}$ and a tangential fwhm of $3.0 \mathrm{~mm}$. The ${ }^{32} \mathrm{Na}$ source used here id.d the ${ }^{18} \mathrm{~F}$ used in brain studies) has positron energies below $600 \mathrm{keV}$ and the blurring caused by the positron range is less than $1 \mathrm{~mm}^{10,11}$. The radial elongation visible in Figure 4 for sources far from the gantry center is due to crystal penetration.

These results can be understood in terms of three factors (Table 3): (1) the detector size, which contributes a triangular PSF with $1.5 \mathrm{~mm}$ fwhm at the center of the gantry, (2) the deviations from $180^{\circ}$ emission, which contributes a nearly Gaussian PSF with $1.3 \mathrm{~mm}$ fwhm, (3) the range of the positrons in tissue, which we approximate here $\left({ }^{18} \mathrm{~F},{ }^{22} \mathrm{Na}\right)$ to be $0.5 \mathrm{~mm}$. The resulting detector pair resolution is $2.0 \mathrm{~mm} \mathrm{fwhm}$, which is degraded by about $45 \%$ in the reconstructed image to 2.9 $\mathrm{mm}$ fwhm. We expect an improvement in the reconstructed image resolution when convolutions are performed on $0.79 \mathrm{~mm}$ projection bins.

\section{TABLE 2: RESULTS}

Timing resolution (first photoelectron) Coincidence window ( $90 \%$ acceptance)

Photopeak resolution

Detector pair resolution

Reconstructed Image resolution:

$$
\text { at } 0 \mathrm{~cm}
$$

at $8 \mathrm{~cm}$
5 nsec fwhm

9 nsec

$20-30 \%$ fwhm

$2.4 \mathrm{~mm}$ fwhm

$2.9 \mathrm{~mm}$ fwhm

$3.0 \times 4.0 \mathrm{~mm}$ fwhm 
Figure 2: Experimental setup for measuring crystal pair resolution by moving a $0.35 \mathrm{~mm}$ diam ${ }^{22} \mathrm{Na}$ wire source at right angles to the line between the crystals.

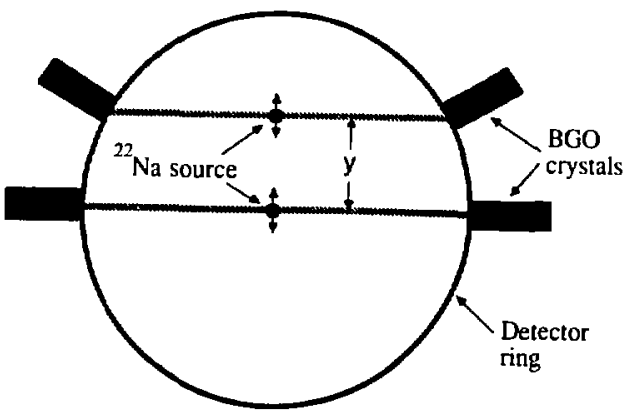

NaL 6610-3963

Figure 3: Coincident event rate recorded between two crystals as a $0.35 \mathrm{~mm}$ diam ${ }^{22} \mathrm{Na}$ wire source is moved in $0.2 \mathrm{~mm}$ steps at right angles to the line between them. At the center of the tomograph $(\mathrm{y}=0 \mathrm{~cm})$, the fwhm is $2.4 \mathrm{~mm}$. At a distance of $y=8.2 \mathrm{~cm}$ from the center, the fwhm is 3.9 mrn. At a distance of $y=$ $12.5 \mathrm{~cm}$ (near the edge of the field), the fwhm is $\mathbf{5 . 0}$ $\mathrm{mm}$ fwhm. Since only photopeak (> $350 \mathrm{keV}$ ) events are selected, this broadening is primarily due to crys-

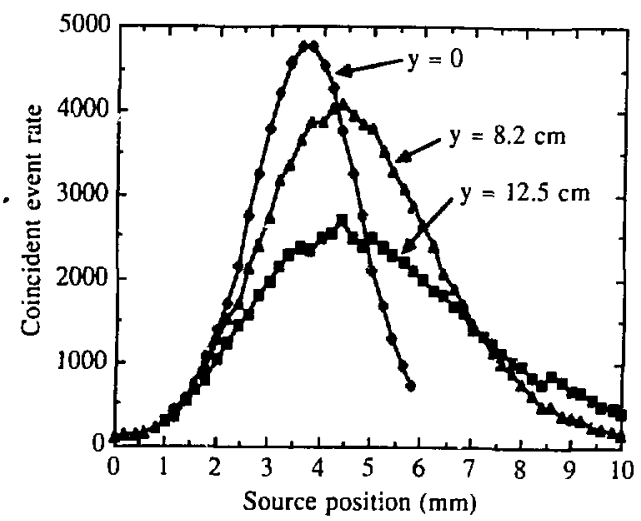
tal penetration. 
Figure 4: Resolution in the reconstructed image of a $0.35 \mathrm{~mm}$ diam ${ }^{22} \mathrm{NA}$ wire as a function of the distance to the center of the tomograph. Clam motion was used with $1.57 \mathrm{~mm}$ projection bins, and the image was a superposition of the clam open and clam shut backprojections.

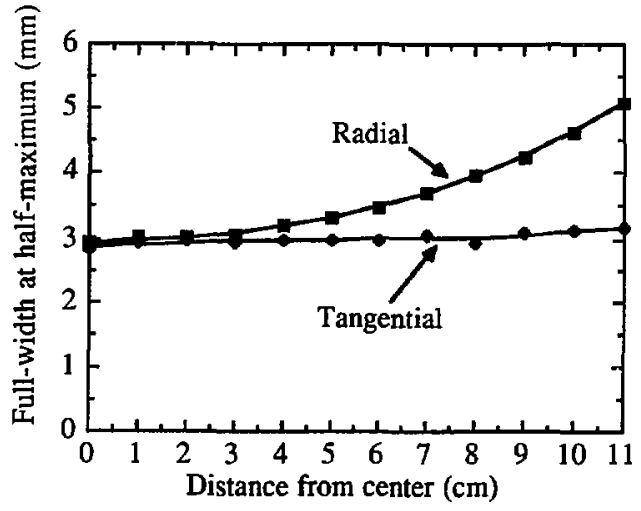

I81 $8610-3948$

TABLE 3: ANTICIPATED RESOLUTION (fwhm)

FACTOR

CONTRIBUTION

Detector size

Deviations from $180^{\circ}$

Positron Range $\left({ }^{18} \mathrm{~F}\right)$

Detector pair sesolution

(added in quadrature)
$1.5 \mathrm{~mm}$ (triangular)

$1.3 \mathrm{~mm}$ (Gaussian)

$0.5 \mathrm{~mm} \dagger$ (sharply peaked)

$2.0 \mathrm{~mm}$

Reconstructed Image Resolution $2.6 \mathrm{~mm}$ ( $30 \%$ increase due to filter)

†Calculated as $\mathbf{2 . 3 5}$ times the measured rms deviation from Reference 10. 
Figure $5 \mathrm{~A}$ is a sketch of the pie-shaped hot spot phantom previously reported. ${ }^{12}$ Figure $5 B$ slows an image of 200 million ${ }^{18} \mathrm{~F}$ events taken with the Donner 280 Crystal Positron Tomograph using clam sampling and reconstructed with $2.5 \mathrm{~mm}$ projection bins. Figure $5 \mathrm{C}$ shows an image of 35 million ${ }^{68} \mathrm{Ga}$ events taken with the Donner 600 Crystal Positron Tomograph in stationary mode and reconstructed with $1.57 \mathrm{~mm}$ projection bins. The improvement resulting from reducing the crystal size from $9.5 \mathrm{~mm}$ to $3 \mathrm{~mm}$ is quite evident.

$12 \mathrm{~mm} \mathrm{c}-\mathrm{c}$

$3.0 \mathrm{~mm}$ diam

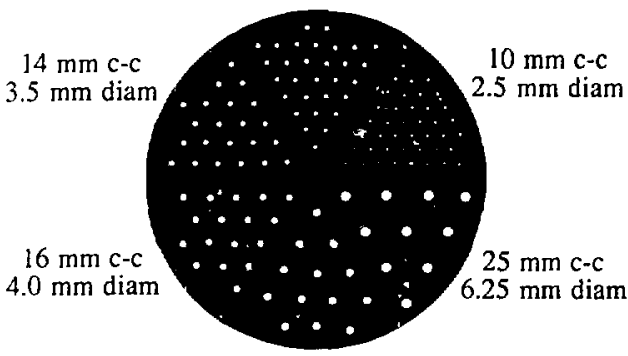

$20 \mathrm{~mm} \mathrm{c}-\mathrm{c}$ $5.0 \mathrm{~mm}$ diam

A

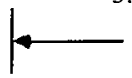

$200 \mathrm{~mm}$

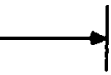

IEL $8610-4031$

Figure 5: A- Sketch of hot spot phantom fabricated by drilling channels in a solid cylinder of plexiglass.

$\mathrm{B}$ - Image of 200 million ${ }^{18} \mathrm{~F}$ events taken with the Donner 280 Crystal Positron Tomograph using clam sampling and reconstructed with $2.5 \mathrm{~mm}$ projection bins. C- Image of 35 million ${ }^{68} \mathrm{Ga}$ events taken with the Donner 600 Crystal Positron Tomograph in stationary mode and reconstructed with $1.57 \mathrm{~mm}$ projection bins
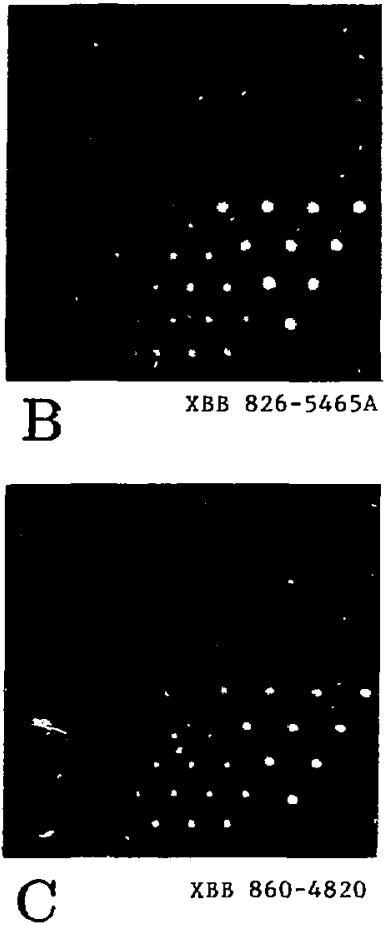
A new phantom was designed (Figure 6A) to challenge the resolution of the new tomograph and contains 6 sectors having circular channels whose center-tocenter spacing is $12 \mathrm{~mm}, 10 \mathrm{~mm}, 8 \mathrm{~mm}, 6 \mathrm{~mm}, 5 \mathrm{~mm}$, and $4 \mathrm{~mm}$. In each case the diameter of the channel is one-fourth the center-to-center spacing. Figure $6 \mathrm{~B}$ shows an image of 120 million ${ }^{18} \mathrm{~F}$ events taken with the Donner 600 Crystal Positron Tomograph using clam sampling and reconstructed with $1.57 \mathrm{~mm}$ projection bins.

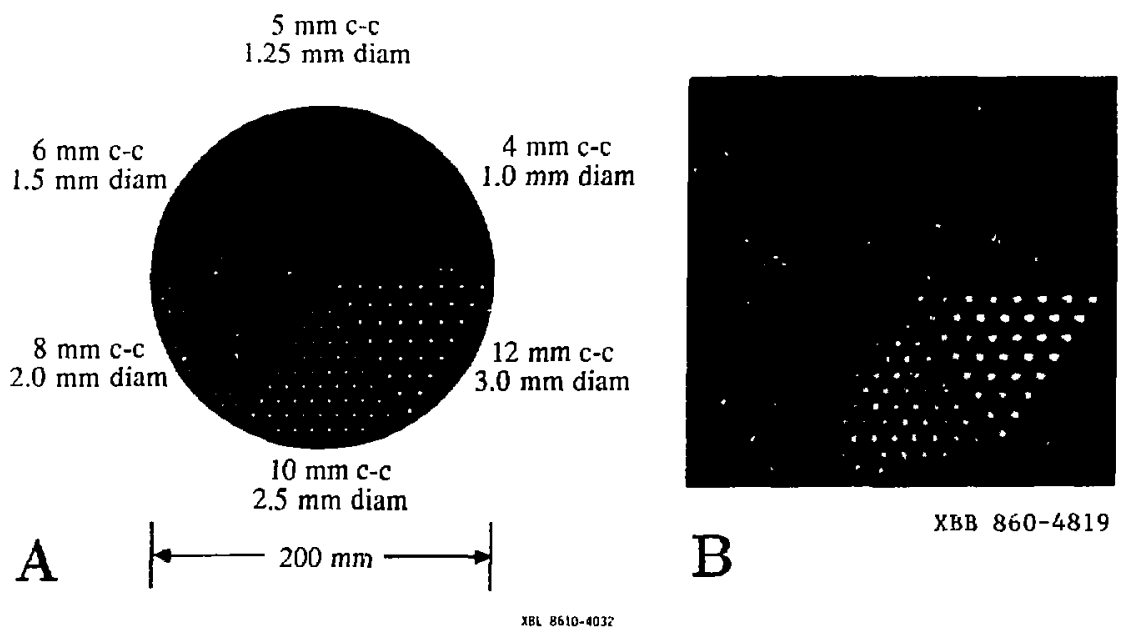

Figure 6: A- Sketch of high resolution hot spot phantom fabricated by drilling channels in a solid cylinder of plexiglass.

$\mathrm{B}$ - Image of 120 million ${ }^{18} \mathrm{~F}$ events taken with the Donner 600 Crystal Positron Tomograph using clam sampling and reconstruced with $1.57 \mathrm{~mm}$ projection bins. 


\section{Future Developments}

We are in the process of verifying the mechanical accuracy of the clam motion and programming an array processor for the rapid reconstruction of clam-sampled projection data in $0.79 \mathrm{~mm}$ bins. The use of position-sensitive silicon photodiodes in future tomograph designs would provide improved sensitivity through the use of multiple layers of detectors and the ability to correct for crystal penetration by measuring the depth of interaction.

\section{Discussion and Conclusions}

In conclusion, these results show that $3 \mathrm{~mm}$ BGO crystals can improve the resolution in positron tomography by a substantial factor. This measured crystal pair resolution of $2.4 \mathrm{~mm}$ fwhm and the reconstructed image resolution of $2.9 \mathrm{~mm}$ fwhm at the center of the tomograph are in good agreement with expected values. The most serious limitation of the detector design is that only a single section can be imaged.

\section{Acknowledgements}

We thank B. Leskovar, C.C. Low, B. Turko, and G. Zizka for contributions to the design and development of the front-end electronics, John Berkowitz for the mechanical design of the gantry, $\mathbf{X}$. Xie for contributions to detector design, and $K$. Brennan, K. Bristol, M. Colina, J. Gurule, B. Jarrett, J. Joseph, M. Morimoto, C. Cullander, and J. Twitchell for numerous contributions.

*This work was suf, orted in part by the Director, Office of Energy Research, Office of Health and Environmental Research of the U.S. Department of Energy, under Contract No. DE-AC03-76SF00098, and in part by Public Health Service Grant Nos. P01 HL25840 and RO1 CA38086 awarded by the National Heart Lung and Blood and National Cancer Institutes, Department of Health and Human Services.

Reference to a company or product name does not imply approval or recommendation of the product by the University of California or the U.S. Department of Energy tu the exclusion of others that may be suitable. 


\section{References}

1. Burnham CA, Bradshaw J, Kaufman D, et al: Design of cylindrical shaped scintillation camera for positron tomographs. IEEE Trans Nucl Sci NS-32: 889-893, 1985

2. Tomitani $\mathrm{T}$, Nohara $\mathrm{N}$, Morayama $\mathrm{H}$, et al: Development of a high resolution positron CT for animal studies. IEEE Trans Nucl Sci NS-32: 822-825, 1985

3. Computer Technology and Imaging, Inc. Knoxville, Tennessee, MODEL PT 931 ECAT Scanner System Description.

4. Darenzo SE, Budinger TF, and Vuletich $T$ : High resolution positron emission tomography usine narrow bismuth germenate crystals and individua! photosensors. IEEE Trans Nucl Sci NS-30: 665-670, 1983

5. Huesman RH, Derenzo SE and Bıdinger TF: $A$ two-position sampling scheme for positron emission tomograpiy. In Nuclear Medicine and Biology, Raynaud C, ed., Pergammon Press, New York, Vol I, pp 542-545, 1983.

6. Cahoon JL, Huesman RH, Derenzo SE, et al: The electronics for the Donner, high resolution 600-crystal positron tomograph. IEEE Trans Nucl Sci NS-33: $570-574,1986$

7. Turko BT, Zizka G, Lo CC, Leskovar B, Cahoon JL, Huesman RH, Derenzo $\mathrm{SE}$, Geyer $\mathrm{AB}$, and Budinger TF: Scintillation photon detection and avent selection in high resolution positron emission tomography. Submitted to IEEE Trans Nucl Sci, 1986

8. Fabricated by Isotope Products Laboratories, Burbank, CA

9. Ramachandran GN and Lakshminarayanan AV: Three-dimensional reconstruction from radiographs and electron micrographs: application of convolutions instead of Fourier Transforms. Proc Nat Acad Sci US 68: 2236-2240, 1971

10. Derenzo, SE: Precision measurement of annihilation point spread distributions for medically important positron emitters. In: Positron Annihilation, Hasiguti RR and Fujiwara K, eds, pp 819-823, The Japan Institute of Metals, Sendai, Japan, 1979

11. Derenzo SE: Mathematical removal of positron range blurring in high resolution tomography. IEEE Trans Nucl Sci NS-33: 565-569, 1986

12. Derenzo SE, Budinger TF, Huesman, RH, Cahonn IL and Vuletich T: Imaging properties of a positron tomograph with 280 BGO crystals. IEEE Trans Nucl Sci NS-28: 81-89, 1.981 


\section{DISCLAIMER}

This report was prepared as an aecount of work sponsored by an agency of the United States Government. Neither the United States Government nor any agency thereof, nor any of their employees, makes any warranty, express or implied, or assumes any legal liability or responsibility for the accuracy, completeness, or usclalness of any information, apparatus. product, or process disclosed, or represents that its use would not infringe privately owned rights. Reference herein to any specific commercial product, process, or service by trade name, trademark, manufacturer, or otherwise does not necessarily constitute or imply its endorsement, recommendation, or favoring by the United States Government or any agency thereor. The views and opinions of authors expressed her in do not necessarily state or reflect those of the United States Government or any agency thereof. 